\title{
Synthesis of Small Gold Nanorods and Their Subsequent Functionalization with Hairpin Single Stranded DNA
}

\author{
Zendesha S. Mbalaha, Paul R. Edwards, ${ }^{\circledR}$ David J.S. Birch, and Yu Chen*(i) \\ Department of Physics, Scottish Universities Physics Alliance, University of Strathclyde, 107 Rottenrow, Glasgow G4 0NG, U.K.
}

\section{Supporting Information}

\begin{abstract}
Small gold nanorods have a significantly large absorption/scattering ratio and are especially beneficial in exploiting photothermal effects, for example in photothermal therapy and remote drug release. This work systematically investigates the influence of growth conditions on the size, growth yield, and stability of small gold nanorods. The silver-assisted seed-mediated growth method was optimized to synthesize stable small gold nanorods with a high growth yield $(>85 \%)$. Further study on the influence of silver ions on the growth facilitates the growth of small gold nanorods with tunable longitudinal surface plasmon resonance from 613 to $912 \mathrm{~nm}$, with average dimensions of $13-25 \mathrm{~nm}$ in length and 5-6 nm in diameter. Moreover, the small gold nanorods were successfully functionalized with thiol-modified hairpin oligonucleotides (hpDNA) labeled with Cy5. Fluorescence intensity measurements show an increase in the presence of target DNA and an enhanced signal/background ratio when the longitudinal surface plasmon resonance of small gold nanorods overlaps with the excitation and emission wavelength of Cy5. This coincides with a reduced fluorescence lifetime of $\mathrm{Cy} 5$ in the hairpin structure, indicating surface plasmon resonance-enhanced energy transfer to the small gold nanorods. This study may provide insight on the synthesis and functionalization of small gold nanorods in biomedical sensing and therapy.
\end{abstract}

\section{INTRODUCTION}

Gold nanorods have been demonstrated as promising agents for various biomedical applications including cancer diagnosis and treatment. ${ }^{1-3}$ The choice of gold nanorods stems from their unique optical properties arising from localized surface plasmon resonance. ${ }^{4,5}$ The longitudinal surface plasmon mode of gold nanorods depends on the particle aspect ratio ${ }^{4-6}$ and can thus be tuned from the visible to the near infrared (NIR) region. This shape-dependent optoelectronic property manifests itself in various processes such as strong surface-enhanced Raman scattering, two-photon luminescence, surface plasmon enhanced energy transfer, and photothermal effects. ${ }^{7-13}$ These combined with low toxicity, water solubility, and biocompatibility, have made gold nanorods a versatile nanomaterial with demonstrated applications in various areas including biological sensing, imaging, drug delivery, and cancer therapy. ${ }^{14-20}$

Localized surface plasmon resonance results in enhanced optical absorption and scattering. Gold nanorods with a longitudinal surface plasmon mode in the NIR region that matches the biological window are ideal agents for biomedical sensing, imaging, and photothermal therapy in treating cancer. Functionalized gold nanorods have been demonstrated as useful probes in energy transfer studies for understanding molecular interactions, recognition of analytes, and binding sites in intracellular environments. ${ }^{21,22}$ For example, the energy transfer process from the fluorophore (donor) to gold nanorods (acceptor) has been exploited to good effect to understand the molecular interactions between proteins, DNA, mRNA, and fluorescence lifetime-based sensing of bio- analytes. $^{21-23}$ In addition, Wei et al. demonstrated the assembly of fluorophore-labeled hairpin DNA (hpDNA) on the large gold nanorod structure for detecting mRNA in a homogenous solution. ${ }^{24}$ hpDNA has been utilized in various biological applications because of its increased sensitivity and specificity for target recognition. ${ }^{24,25}$

Small gold nanorods are especially beneficial for applications exploiting the photothermal effect, ${ }^{26,27}$ as they have a higher absorption to scattering ratio than that of larger gold nanorods, therefore converting more energy to heat. ${ }^{20}$ In addition, small size gold nanorods have a large surface area to volume ratio, high cellular uptake, high clearance rate from the liver, spleen, and the kidney, and ease of subcellular accessibility. ${ }^{28-36}$ Furthermore, the efficient fluorescence quenching abilities of small gold nanorods render them good quenchers of background signals in fluorescence detection techniques. ${ }^{37}$ Small gold nanorods are therefore ideal agents for photothermal therapy, diagnostic, biosensing, and drug delivery. ${ }^{5}$

Most of the relevant works so far have used gold nanorods synthesized via a seed-mediated growth method developed by Murphy's and El-Sayed's groups. ${ }^{38,39}$ The gold nanorods thus synthesized are typically over $50 \mathrm{~nm}$ in length and $11-15 \mathrm{~nm}$ in diameter. ${ }^{29,31,35,40}$ Recently, Ali et al. ${ }^{40}$ synthesized small gold nanorods (18-25 $\mathrm{nm}$ in length and $4-5 \mathrm{~nm}$ in diameter) via a seedless growth method by adjusting the $\mathrm{pH}$ and

Received: April 26, 2019

Accepted: July 30, 2019 
concentration of sodium borohydride $\left(\mathrm{NaBH}_{4}\right)$ and hexadecyltrimethylammonium bromide ( $\mathrm{CTAB})$, respectively. Jia et al. $^{26}$ synthesized small gold nanorods with a diameter of less than $10 \mathrm{~nm}$ via the seed-mediated method by simultaneously varying both the amount of seed and $C T A B$ added to the growth solutions to tune the diameter of the gold nanorods. However, synthesis of stable, small gold nanorods with controlled aspect ratios to achieve surface plasmon resonance at selected wavelengths spanning a large wavelength region remains challenging.

Here, we have systematically investigated the influence of growth conditions on the size, growth yield, and stability of small gold nanorods. Stable small gold nanorods of improved yield were achieved by optimizing the amount of seeds added to the growth solution (shown in Figure 1). Small nanorods of

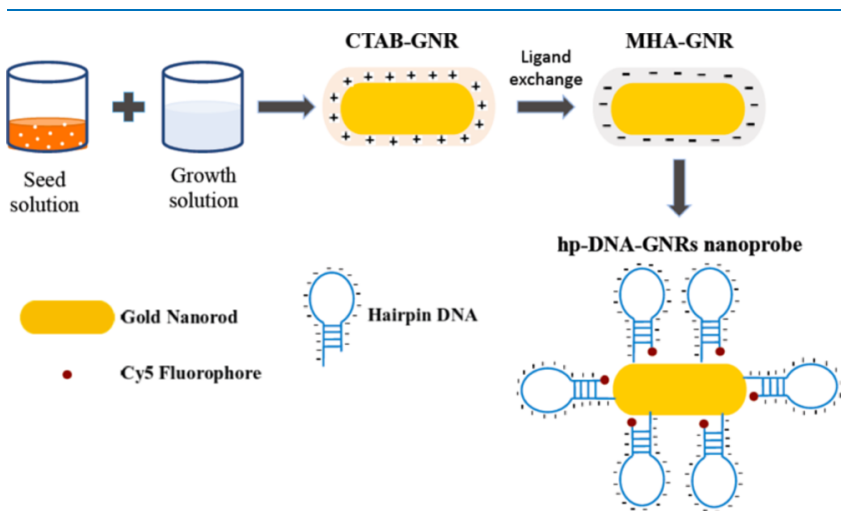

Figure 1. A scheme showing the synthesis and functionalization of small gold nanorods.

varying aspect ratios with longitudinal surface plasmon resonance from 613 to $912 \mathrm{~nm}$ were successfully synthesized by simply varying the amount of silver nitrate in the growth solution. Specifically, stable short small gold nanorods were synthesized with an average length of $14.2 \mathrm{~nm}$. Moreover, we report for the first time the functionalization of small gold nanorods with thiolated Cy5-labeled single strand hpDNA via the ligand exchange and salt aging process. This demonstrates the possibility of assembling thiolated aptamer and other DNA structures onto small gold nanorods. Therefore, this work should facilitate the development of small gold nanorod-based agents for enhanced performance in targeted analyte sensing, biomolecular labeling, photothermal therapy, and drug delivery.

\section{RESULTS AND DISCUSSION}

2.1. Influence of Seeds and CTAB on the Size of Small Gold Nanorods. The silver-assisted seed-mediated growth method was used to synthesize small gold nanorods due to its flexibility and ease of control over particle size and shape. To study the influence of seeds and $\mathrm{CTAB}$ on the size of gold nanorods, we adopted the reported silver-assisted seedmediated growth protocol ${ }^{26}$ to produce three samples coded as $\mathrm{G}_{9} \mathrm{~S}_{1}, \mathrm{G}_{8} \mathrm{~S}_{2}$, and $\mathrm{G}_{6} \mathrm{~S}_{4}$ as described in Section 4.2. Figure 2 shows the scanning electron microscopy (SEM) images and extinction spectra of these samples. It was found that the gold nanorods had an average length of less than $17 \mathrm{~nm}$ and diameter below $6 \mathrm{~nm}$ as shown in Table 1. The distribution of sizes of small gold nanorods for these samples are shown in the Supporting Information (Figure S1). Both the length and
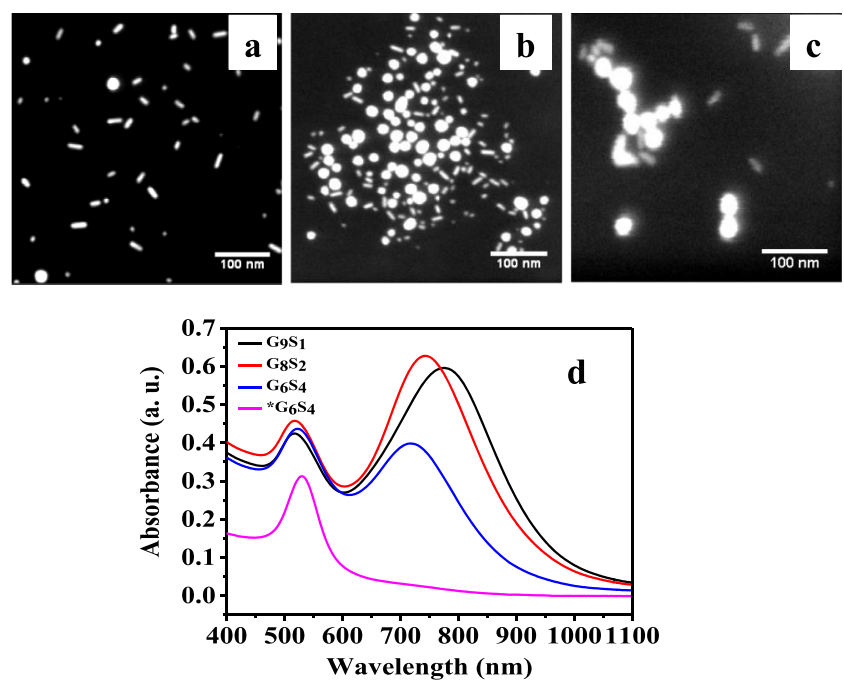

Figure 2. SEM of small gold nanorods prepared by varying the ratio of growth solution (G) to seed solution (S); (a) $\mathrm{G}_{9} \mathrm{~S}_{1}$, (b) $\mathrm{G}_{8} \mathrm{~S}_{2}$, (c) $\mathrm{G}_{6} \mathrm{~S}_{4}$, and (d) UV-vis extinction spectra.

Table 1. Size Distribution, Longitudinal Mode Wavelength, and Growth Yield of Small Gold Nanorods at Varying Seeds to CTAB Solution Ratios

\begin{tabular}{ccccc} 
samples & LSPR $(\mathrm{nm})$ & length $(\mathrm{nm})$ & diameter $(\mathrm{nm})$ & yield (\%) \\
$\mathrm{G}_{9} \mathrm{~S}_{1}$ & 776 & $16.3 \pm 2.9$ & $6.0 \pm 1.4$ & 69 \\
$\mathrm{G}_{8} \mathrm{~S}_{2}$ & 744 & $14.9 \pm 2.9$ & $6.0 \pm 1.2$ & 51 \\
$\mathrm{G}_{6} \mathrm{~S}_{4}$ & 717 & $13.1 \pm 2.8$ & $5.0 \pm 1.4$ & 12 \\
\hline
\end{tabular}

diameter decrease by increasing the amount of seeds added in the growth solution. The decrease in size as the seed amount increases is not surprising as increased nucleation sites results in a reduced amount of gold atoms available to each nanorod. $^{41,42}$ Similarly, the aspect ratio decreases as the amount of seeds in the growth solution increases due to the shortening of length ${ }^{26,43}$ that leads to a shift in the longitudinal surface plasmon resonance peaks from 776 , to 744 , to $717 \mathrm{~nm}$ for $\mathrm{G}_{9} \mathrm{~S}_{1}, \mathrm{G}_{8} \mathrm{~S}_{2}$, and $\mathrm{G}_{6} \mathrm{~S}_{4}$, respectively.

The growth yield of small gold nanorods (number of nanorods/number of total particles) reached $69 \%$ in $\mathrm{G}_{9} \mathrm{~S}_{1}$ as shown in Table 1 . It decreases dramatically to $12 \%$ in $\mathrm{G}_{6} \mathrm{~S}_{4}$ as the seed to CTAB ratio in the growth solution increases. This could be due to a reduced amount of CTAB surfactant selectively binding to the sides of each nanorod, resulting in a less anisotropic growth and generation of more nanospheres. Previous reports indicated that more byproducts of nanospheres were formed than gold nanorods as the amount of seed solution in the final growth mixture increased. ${ }^{26,40,44}$ Moreover, $\mathrm{G}_{6} \mathrm{~S}_{4}$ was found not to be stable; Figure $2 \mathrm{~d}$ shows that the UV-vis extinction spectrum changed from the initial two surface plasmon bands $\left(\mathrm{G}_{6} \mathrm{~S}_{4}\right)$ to one peak $\left({ }^{*} \mathrm{G}_{6} \mathrm{~S}_{4}\right)$ after 24 days of synthesis, indicative of the transformation of gold nanorods to gold nanospheres. This implies the limitation of synthesizing short smaller gold nanorods by merely increasing the seed to growth solution ratio.

2.2. Influence of Silver Nitrate on the Growth of Small Gold Nanorods. Silver ions play a vital role in the formation of gold nanorods and impact on their aspect ratios and growth yields. Silver ions form complexes with CTAB to regulate the structural evolution of gold seeds to the rod-like shape and improve the nanorod yield. ${ }^{45,46}$ To investigate the 
influence of silver ions on the yield and morphology of small gold nanorods, we varied the amount of $\mathrm{AgNO}_{3}$ based on the recipe for sample $\mathrm{G}_{9} \mathrm{~S}_{1}$. Specifically, the amount of silver nitrate used in the growth solution was varied from 150 to $20 \mu \mathrm{L}$ in nine samples, while other reagents were unchanged as detailed in 4.3.

Figure 3 shows typical SEM images taken from these nine samples. Average length, diameter, and yield of nanorods
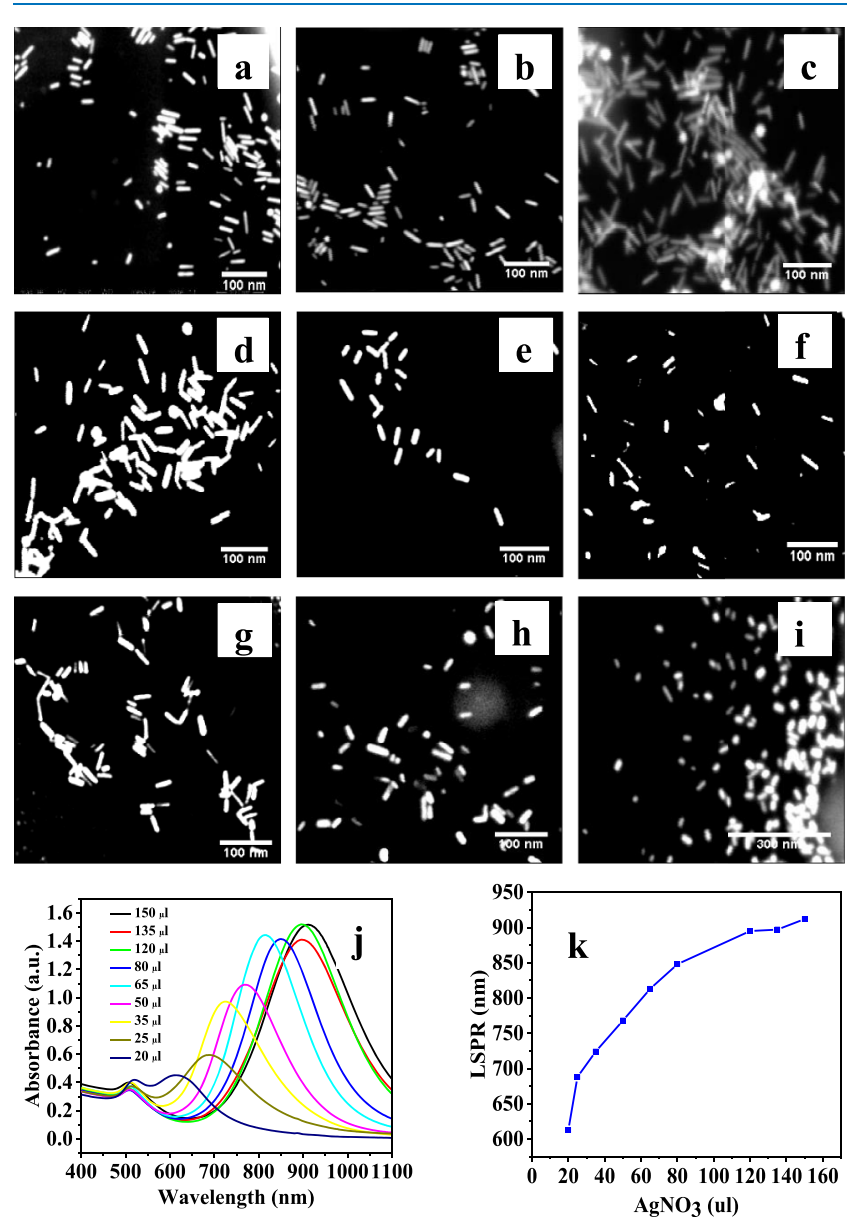

Figure 3. SEM images of small gold nanorods prepared by varying the amount of silver nitrate in the growth solution; (a) $\mathrm{G}_{9} \mathrm{~S}_{1}-150 \mu \mathrm{L}$ $\mathrm{AgNO}_{3}$; (b) $\mathrm{G}_{9} \mathrm{~S}_{1}-135 \mu \mathrm{L} \quad \mathrm{AgNO}_{3}$; (c) $\mathrm{G}_{9} \mathrm{~S}_{1}-120 \mu \mathrm{L} \quad \mathrm{AgNO}_{3}$; (d) $\mathrm{G}_{9} \mathrm{~S}_{1}-80 \mu \mathrm{L} \mathrm{AgNO}_{3}$; (e) $\mathrm{G}_{9} \mathrm{~S}_{1}-65 \mu \mathrm{L} \mathrm{AgNO}_{3}$; (f) $\mathrm{G}_{9} \mathrm{~S}_{1}-50 \mu \mathrm{L} \mathrm{AgNO}_{3}$; (g) $\mathrm{G}_{9} \mathrm{~S}_{1}-35 \mu \mathrm{L} \mathrm{AgNO}_{3}$; (h) $\mathrm{G}_{9} \mathrm{~S}_{1}-25 \mu \mathrm{L} \mathrm{AgNO}_{3}$; (i) $\mathrm{G}_{9} \mathrm{~S}_{1}-20 \mu \mathrm{L}$ $\mathrm{AgNO}_{3}$; (j) UV-vis extinction spectra of the samples; (k) relationship between the amount of silver nitrate in the growth solution and longitudinal surface plasmon resonance wavelength.

derived from SEM images are listed in Table 2. The distribution of the sizes of the synthesized small gold nanorods are shown in the Supporting Information (Figure S2). It can be seen that the length of the nanorods decreases from about 25 to $14 \mathrm{~nm}$ as silver nitrate decreased from 150 to $20 \mu \mathrm{L}$. The diameter is less sensitive to the amount of silver nitrate, varying slightly between 5 and $6.5 \mathrm{~nm}$. Silver ions were found to promote the anisotropic growth to a nanorod shape although the specific role of silver ions in the synthesis is still not fully understood $^{38,39,47}$ The three mechanisms proposed so far include under-potential deposition to favor growth on longitudinal facets, face-specific capping to block specific facets, and modification of CTAB micelle formation through
Table 2. Size Distribution of the Wavelength of the Longitudinal Absorption, Average Length, Average Diameter, and Nanorod Yield for Different Amounts of Silver Nitrate $\left(\mathrm{AgNO}_{3}, 0.01 \mathrm{M}\right)$

\begin{tabular}{ccccc}
$\mathrm{AgNO}_{3}(\mu \mathrm{L})$ & LSPR $(\mathrm{nm})$ & length $(\mathrm{nm})$ & diameter $(\mathrm{nm})$ & yield (\%) \\
150 & 912 & $25.7 \pm 5.5$ & $5.9 \pm 1.1$ & 85.28 \\
135 & 897 & $25.0 \pm 5.6$ & $6.3 \pm 1.4$ & 90.95 \\
120 & 895 & $24.6 \pm 4.7$ & $5.8 \pm 1.0$ & 87.20 \\
80 & 848 & $23.3 \pm 4.2$ & $5.7 \pm 1.4$ & 97.94 \\
65 & 813 & $23.9 \pm 3.9$ & $6.6 \pm 1.1$ & 94.33 \\
50 & 768 & $22.8 \pm 4.4$ & $5.5 \pm 1.3$ & 92.11 \\
35 & 724 & $22.0 \pm 4.5$ & $5.6 \pm 1.3$ & 98.94 \\
25 & 688 & $18.3 \pm 4.6$ & $5.2 \pm 1.3$ & 93.10 \\
20 & 613 & $14.2 \pm 4.0$ & $5.1 \pm 1.5$ & 90.60 \\
\hline
\end{tabular}

silver-bromide integrations. ${ }^{39,48}$ The dependence of the morphology of small gold nanorods on the amount of silver ions observed in this work is in line with a previous study on conventional (large) gold nanorods. Moreover, high yields of nanorod formation $(>85 \%)$ were found for all the nine samples.

Figure $3 \mathrm{j}$ depicts the UV-vis extinction spectra taken from the nine samples. A significant blue-shift in the longitudinal surface plasmon resonance from 912 to $613 \mathrm{~nm}$ was observed as the amount of silver nitrate decreases from 150 to $20 \mu \mathrm{L}$. A near linear relationship was found between the wavelength of the longitudinal surface plasmon mode $(688$ to $850 \mathrm{~nm})$ and silver nitrate amount $(25$ to $80 \mu \mathrm{L})$, as depicted in Figure $3 \mathrm{k}$. At a higher silver nitrate concentration $(>100 \mu \mathrm{L})$, the surface plasmon resonance remains around $900 \mathrm{~nm}$. These findings are consistent with previous reports on the growth of large gold nanorods. ${ }^{39,41}$ This could imply that the influence of silver ions on the longitudinal surface plasmon mode of small gold nanorods is similar to conventional gold nanorods. The average lengths of nanorods were also found to fluctuate between 24 and $25 \mathrm{~nm}$. Tong et al. recently found that the control of silver ions on the aspect ratio of conventional gold nanorods occurs during the symmetry breaking period and the length of the final nanorods formed is dependent on the available gold atom concentration. ${ }^{45}$ Therefore, the unchanged length at a higher silver nitrate concentration suggests the depletion of the available gold atoms.

2.3. Spectroscopic Study of Small Gold NanorodBased Nanoprobes. Functionalization of gold nanoparticles is of importance for their biological and biomedical applications. Recently, we have reported a new RNA nanoprobe based on functionalized conventional GNRs with single strand DNA (ssDNA) in a hairpin structure, and the influence of the hairpin structure and packing density on the performance of nanoprobes. ${ }^{24}$ The ssDNA hairpin contains a sequence complementary to the target mRNA. The fluorophores are quenched in the absence of target mRNA. In the presence of the target mRNA, the hairpin opens as a result of RNA-DNA base-paring, and fluorescence is recovered or even enhanced with optimal hairpin design. Here, we employed a similar synthetic approach to functionalize small gold nanorods with ssDNA labeled with Cy5. The fluorescence of Cy5 in the hairpin structure is quenched via energy transfer from $\mathrm{Cy} 5$ to the gold nanorod, and the energy transfer is prominent when the surface plasmon resonance of gold nanorods is matched to the emission of the fluorophore. To investigate the influence of surface plasmon resonance on the performance of nanoprobes, 
we selected two types of small gold nanorods with different longitudinal surface plasmon resonances. The longitudinal surface plasmon resonance of the first sample is away from the emission spectrum of $\mathrm{Cy} 5$ while the second sample matches the emission spectrum of Cy5. The recipe for the $\mathrm{G}_{9} \mathrm{~S}_{1}$ series was adjusted to produce samples $\mathrm{LG}_{18} \mathrm{~S}_{2}$ and $\mathrm{SG}_{18} \mathrm{~S}_{2}$ by scaling up $(2 \times)$ the reagents with different silver nitrate amounts of 83.73 and $15.84 \mu \mathrm{L}$, respectively. The longitudinal surface plasmon resonances of $\mathrm{LG}_{18} \mathrm{~S}_{2}$ and $\mathrm{SG}_{18} \mathrm{~S}_{2}$ were 857 and 661 $\mathrm{nm}$, as shown in Figure 4a,b; their dimensions were $20.58 \pm$ $4.7 \mathrm{~nm}$ by $5.65 \pm 1.1 \mathrm{~nm}$ and $13.13 \pm 2.1 \mathrm{~nm}$ by $4.89 \pm 1.0$ $\mathrm{nm}$, respectively.
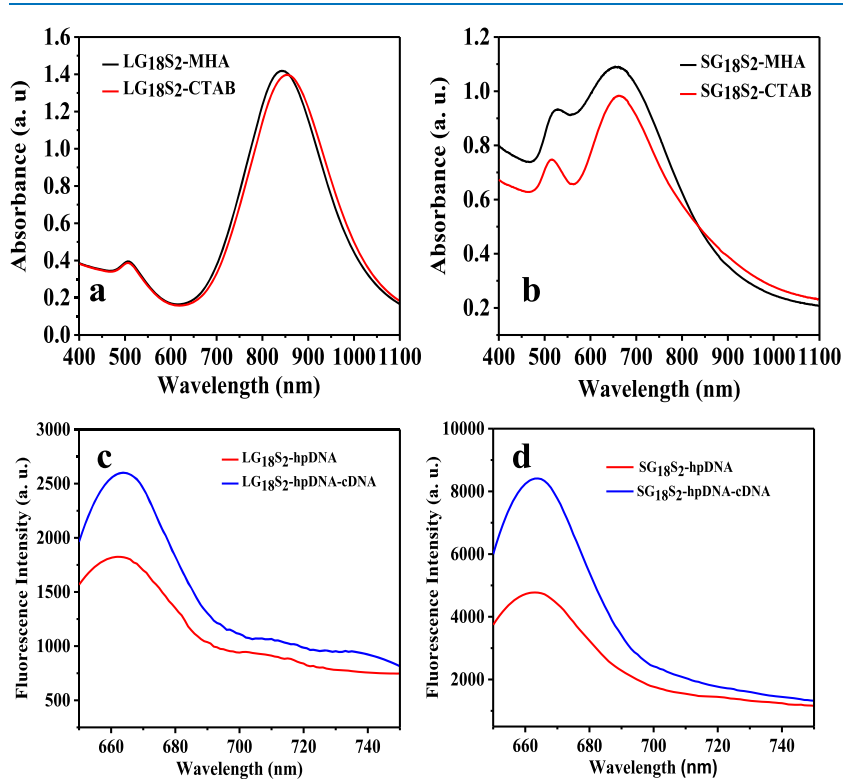

Figure 4. UV-vis extinction spectra of (a) $\mathrm{LG}_{18} \mathrm{~S}_{2}$ and (b) $\mathrm{SG}_{18} \mathrm{~S}_{2}$ before and after ligand exchange; Fluorescence spectra of small gold nanorod nanoprobes (c) $\mathrm{LG}_{18} \mathrm{~S}_{2}$ and (d) $\mathrm{SG}_{18} \mathrm{~S}_{2}$ with/out cDNA.

Gold nanorods coated with CTAB are not suitable for direct bio-applications due to their cytotoxicity to biological tissues. ${ }^{49-51}$ Therefore, the CTAB surfactant on the surface layer of the small gold nanorods was replaced with mercaptohexanoic acid (MHA) via a round-trip phase transfer ligand exchange. ${ }^{24,52}$ Zeta potential measurements showed the change of surface charge from $36.0 \pm 1.1 \mathrm{mV}\left(\mathrm{LG}_{18} \mathrm{~S}_{2}\right)$ and $25.7 \pm 2.7 \mathrm{mV}\left(\mathrm{SG}_{18} \mathrm{~S}_{2}\right)$ prior to ligand exchange, to $-20.1 \pm$ 6.7 and $-12.9 \pm 0.3 \mathrm{mV}$, respectively, after ligand exchange, indicating the replacement of $\mathrm{CTAB}$ by MHA. Figure $4 a, b$ show that the longitudinal surface plasmon resonance bands of $\mathrm{LG}_{18} \mathrm{~S}_{2}$ and $\mathrm{SG}_{18} \mathrm{~S}_{2}$ blue-shifted to 839 and $654 \mathrm{~nm}$ without significantly broadening after the ligand exchange process, indicating a successful ligand exchange with no evident aggregation. $^{24}$
Following ligand exchange, both samples were functionalized with Cy5-labeled hpDNA via a salt aging process as described in Section 4.5. The hpDNA functionalized small gold nanorods were stable with no visible sign of aggregation as shown in the Supporting Information (Figure S3). The functionality of the small gold nanorod-based nanoprobes was evaluated by hybridizing the probes with complementary DNA (cDNA) in a molar ratio of cDNA to GNR-hpDNA 1000:1. The hybridization of cDNA with hpDNA opens the hairpin structure, changing the separation between the Cy5 and gold surface and switching the fluorophore from its initial quenched dark state to an opened bright state.

The fluorescence spectra taken from both samples before and after hybridization are presented in Figure $4 \mathrm{c}, \mathrm{d}$. The emission wavelength of the nanoprobes was found centered at $665 \mathrm{~nm}$ for both the samples that have a larger spectral overlap with the surface plasmon resonance of $\mathrm{SG}_{18} \mathrm{~S}_{2}$-hpDNA and that of $\mathrm{LG}_{18} \mathrm{~S}_{2}$-hpDNA as displayed in the Supporting Information (Figure S4). A significant increase in the fluorescence intensity in the presence of cDNA is apparent in both cases, demonstrating the successful assembly of hpDNA onto small gold nanorods. A larger fluorescence increase (1.8-fold) was observed for $\mathrm{SG}_{18} \mathrm{~S}_{2}$-hpDNA where the longitudinal surface plasmon resonance overlaps with the emission of Cy5. On the other hand, a smaller fluorescence increase (1.4-fold) was observed for $\mathrm{LG}_{18} \mathrm{~S}_{2}$-hpDNA because the longitudinal surface plasmon resonance is away from the excitation and emission of Cy5.

A three-exponential fluorescence lifetime analysis revealed one short lifetime component $\tau_{1}$ arising from the detection of scattered excitation, and two lifetime components, $\tau_{2}$ and $\tau_{3}$, from Cy5 as shown in Table 3. The fluorescence decay curves before and after incubation with cDNA are shown in the Supporting Information (Figure S5). The fluorescence lifetime of the free $\mathrm{Cy} 5$ fluorophore has been reported to be approximately $1.8 \mathrm{~ns}^{53}$ The sub-nanosecond lifetime, $\tau_{2}$, can be attributed to Cy5 near the gold surface in a closed hairpin structure. ${ }^{11,22}$ The longer nanosecond lifetime component, $\tau_{3}$, arises from $\mathrm{Cy} 5$ in an opened hairpin that is relatively unquenched away from the gold surface. It is noted that the average lifetime of $\mathrm{Cy} 5$ in $\mathrm{SG}_{18} \mathrm{~S}_{2}$-hp prior to hybridization $(0.62 \mathrm{~ns})$ is smaller than that in $\mathrm{LG}_{18} \mathrm{~S}_{2}$-hp (0.82 ns), indicating surface plasmon-enhanced energy transfer when the longitudinal mode of $\mathrm{SG}_{18} \mathrm{~S}_{2}$-hp matches with the emission of Cy5. The increase of the average lifetime for both nanoprobes upon hybridization is consistent with the steadystate measurement, confirming the hybridization of the nanoprobe with the target DNA. This demonstrates the potential for employing small gold nanorods for mRNA detection and imaging. Furthermore, the synthesis and functionalization approach reported here can be used to develop functional small gold nanorods for targeted drug delivery and photothermal therapy.

Table 3. Fluorescence Lifetimes of Cy5-hpDNA-Labeled Small Gold Nanorod Nanoprobes before and after Hybridization with cDNA

$\begin{array}{lcccccrrr} & \tau_{1}(\mathrm{~ns}) & B_{1}(\%) & \tau_{2}(\mathrm{~ns}) & B_{2}(\%) & \tau_{3}(\mathrm{~ns}) & B_{3}(\%) & \bar{\tau}(\mathrm{ns}) & \chi^{2} \\ \mathrm{LG}_{18} \mathrm{~S}_{2} \text {-hp } & 0.01 & 86.00 & 0.25 \pm 0.015 & 6.23 & 1.27 \pm 0.015 & 7.77 & 0.82 & 1.01 \\ \mathrm{LG}_{18} \mathrm{~S}_{2} \text {-hp-cDNA } & 0.01 & 75.67 & 0.53 \pm 0.047 & 7.47 & 1.44 \pm 0.015 & 16.86 & 1.16 & 0.93 \\ \mathrm{SG}_{18} \mathrm{~S}_{2} \text {-hp } & 0.01 & 85.52 & 0.21 \pm 0.016 & 8.24 & 1.19 \pm 0.018 & 6.24 & 0.62 & 1.03 \\ \mathrm{SG}_{18} \mathrm{~S}_{2} \text {-hp-cDNA } & 0.01 & 75.96 & 0.38 \pm 0.031 & 9.65 & 1.24 \pm 0.017 & 14.39 & 0.90 & 1.00\end{array}$




\section{CONCLUSIONS}

We have demonstrated a successful synthesis of stable small gold nanorods of various sizes and aspect ratios with tunable longitudinal surface plasmon resonance from 613 to $912 \mathrm{~nm}$ by the silver-assisted seed-mediated growth method. It was found that a decrease in the amount of silver nitrate in the growth solution shortens the length of small gold nanorods. Moreover, small gold nanorods were functionalized with a Cy5-labeled thiol-modified hpDNA. Significant changes in the fluorescence intensities and lifetimes of nanoprobes upon hybridization with cDNA indicate the successful functionalization of small gold nanorods and the capability of small gold nanorod-based probes to detect a potential target including mRNA, microRNA, and ssDNA. Fluorescence intensity measurements show an enhanced signal/background ratio when the longitudinal surface plasmon resonance of small gold nanorods overlaps with the excitation and emission wavelength of Cy5, manifesting the surface plasmon-enhanced energy transfer, and benefiting the optical tunability of gold nanorods. The synthesis and functionalization methods reported in this work should shine light on further developments of functionalized small gold nanorods for applications in biomedical sensing, drug delivery, and photothermal therapy.

\section{EXPERIMENTAL SECTION}

4.1. Materials. All chemicals were used as received without further purification. Chloroauric acid $\left(\mathrm{HAuCl}_{4}, 49 \%\right)$, (CTAB, $99 \%$ ), ascorbic acid (AA), sodium borohydride $\left(\mathrm{NaBH}_{4}\right.$, 99.8\%), silver nitrate $\left(\mathrm{AgNO}_{3}\right)$, dodecanethiol (DDT, 98\%), MHA (99.8\%), acetone (99.9\%), isopropanol (99.5\%), toluene (99.8\%), methanol, thiolated oligonucleotide, and the complementary oligonucleotide were all purchased from Sigma-Aldrich while hydrochloric acid (HCL) was purchased from Fluka.

4.2. Synthesis of Small Gold Nanorods by Varying Seeds and CTAB Concentration. Small gold nanorods were synthesized via the silver-assisted seed-mediated growth method. $^{26}$ To investigate the influence of seeds and CTAB on the growth of small gold nanorods, the growth method was adopted by varying the concentration of seeds in the growth solution. The seed solution was prepared by adding $\mathrm{HAuCl}_{4}$ $(0.01 \mathrm{M}, 0.25 \mathrm{~mL})$ to a solution of CTAB $(0.1 \mathrm{M}, 9.75 \mathrm{~mL})$. Thereafter, a freshly prepared ice-cold $\mathrm{NaBH}_{4}$ solution $(0.01$ $\mathrm{M}, 0.6 \mathrm{~mL}$ ) was added to the mixture and stirred with a magnetic stirrer for 2-3 min until the seed solution turned a dark brown color. The solution was incubated at room temperature for $2 \mathrm{~h}$ for gold seeds' growth before use.

To prepare the growth solutions for three samples $\left(\mathrm{G}_{9} \mathrm{~S}_{1}\right.$, $\mathrm{G}_{8} \mathrm{~S}_{2}$, and $\mathrm{G}_{6} \mathrm{~S}_{4}$ ), various volume of $0.1 \mathrm{M} \mathrm{CTAB}$ solutions (9, 8 , and $6 \mathrm{~mL})$ solutions were made. Then, $\mathrm{HAuCl}_{4}(0.01 \mathrm{M}, 0.5$ $\mathrm{mL}), \mathrm{AgNO}_{3}(0.01 \mathrm{M}, 0.1 \mathrm{~mL})$, and $\mathrm{HCl}(1.0 \mathrm{M}, 0.2 \mathrm{~mL})$ were sequentially added to each solution, respectively. After thorough mixing, AA $(0.1 \mathrm{M}, 0.08 \mathrm{~mL})$ was added to partially reduce gold ions $\left(\mathrm{Au}^{3+}\right.$ to $\left.\mathrm{Au}^{+}\right)$. Stirring continued until the mixture turned colorless. ${ }^{41}$ Finally, 1, 2, and $4 \mathrm{~mL}$ of the seed solution were added to each sample, respectively, and these were then kept at room temperature overnight. The seed solution acts as a template for growth of rod shape in addition to reducing $\mathrm{Au}^{+}$to $\mathrm{Au}^{0}{ }^{39}$ The chemicals used for preparing $\mathrm{G}_{9} \mathrm{~S}_{1}, \mathrm{G}_{8} \mathrm{~S}_{2}$, and $\mathrm{G}_{6} \mathrm{~S}_{4}$ are listed in Table 4.

4.3. Varying Silver Nitrate to the Growth of Small Gold Nanorods. To adjust the aspect ratio of the gold
Table 4. Chemicals Used for Preparing $G_{9} S_{1}, G_{8} S_{2}$, and $G_{6} S_{4}$

\begin{tabular}{llll}
\multicolumn{1}{c}{ sample } & $\mathrm{G}_{9} \mathrm{~S}_{1}$ & $\mathrm{G}_{8} \mathrm{~S}_{2}$ & $\mathrm{G}_{6} \mathrm{~S}_{4}$ \\
$\mathrm{HAuCl}_{4}(0.01 \mathrm{M} ; \mathrm{mL})$ & 0.5 & 0.5 & 0.5 \\
$\mathrm{CTAB}(0.1 \mathrm{M} ; \mathrm{mL})$ & 9.0 & 8.0 & 6.0 \\
$\mathrm{AgNO}_{3}(0.01 \mathrm{M} ; \mathrm{mL})$ & 0.1 & 0.1 & 0.1 \\
$\mathrm{HCl}(1.0 \mathrm{M} ; \mathrm{mL})$ & 0.2 & 0.2 & 0.2 \\
$\mathrm{AA}(0.1 \mathrm{M} ; \mathrm{mL})$ & 0.08 & 0.08 & 0.08 \\
gold seeds $(\mathrm{mL})$ & 1 & 2 & 4 \\
\hline
\end{tabular}

nanorods, we modified the optimized recipe of $\mathrm{G}_{9} \mathrm{~S}_{1}$ by varying the amount of $\mathrm{AgNO}_{3}$ added in the growth solution. The growth solution was prepared by adding $\mathrm{HAuCl}_{4}(0.01 \mathrm{M}, 0.5$ $\mathrm{mL})$ to CTAB $(0.1 \mathrm{M}, 9 \mathrm{~mL})$ in nine separate samples. To each solution, varying amount of $\mathrm{AgNO}_{3}(150,135,120,80$, $65,50,35,25$, and $20 \mu \mathrm{L}$ of $0.01 \mathrm{M}$ ) were added, respectively, followed by adding the same amount of $\mathrm{HCl}(1.0 \mathrm{M}, 0.2 \mathrm{~mL})$ and AA (0.1 M, 0.08 mL). After stirring for a few minutes, 1 $\mathrm{mL}$ of the gold seeds, produced under same conditions as described in Section 4.2, was added to each solution and kept at room temperature overnight. The samples thus made were labeled as $\mathrm{G}_{9} \mathrm{~S}_{1}-150, \mathrm{G}_{9} \mathrm{~S}_{1}-135, \mathrm{G}_{9} \mathrm{~S}_{1}-120, \mathrm{G}_{9} \mathrm{~S}_{1}-80, \mathrm{G}_{9} \mathrm{~S}_{1}-65$, $\mathrm{G}_{9} \mathrm{~S}_{1}-50, \mathrm{G}_{9} \mathrm{~S}_{1}-35, \mathrm{G}_{9} \mathrm{~S}_{1}-25$, and $\mathrm{G}_{9} \mathrm{~S}_{1}-20$. The chemicals used for preparing the growth solution to investigate the influence of silver nitrate on the size of small gold nanorods are shown in the Supporting Information Table S1.

4.4. Ligand Exchange of Short Small Gold Nanorods. The CTAB surfactant on the surface of as-synthesized gold nanorods is not bio-friendly and prevents the direct assembly of hpDNA to the gold surface, therefore ligand exchange was performed to replace the CTAB surfactant via ligand exchange. ${ }^{24}$ We performed ligand exchange for two samples $\left(\mathrm{LG}_{18} \mathrm{~S}_{2}\right.$ and $\left.\mathrm{SG}_{18} \mathrm{~S}_{2}\right)$ using round-trip phase transfer ligand exchange protocol previously reported in the literature. ${ }^{24,52}$ Specifically, the CTAB surfactant on the surface of small gold nanorods was replaced by MHA. The pellets of ligandexchanged small gold nanorods were suspended in TBE $(\mathrm{pH}$ 8.5, $50 \mu \mathrm{L}$ ) buffer.

4.5. Functionalization of Small Gold Nanorods. Following the ligand exchange, the gold nanorods were functionalized with thiolated hpDNA labeled with Cy5 according to a previous protocol ${ }^{24}$ described in Figure 1 . hpDNA is composed of Cy5 label located at the $5^{\prime}$ end and the thiol group at the $3^{\prime}$ end (5'-Cy5-CTGACTTG GTG AAG CTA ACG TTG AG CAAGTCAG-AA- $\left.\left(\mathrm{CH}_{2}\right)_{6}-\mathrm{HS}-3^{\prime}\right)$. The thiolated hpDNA $\left(10^{5} \mathrm{nM}, 10 \mu \mathrm{L}\right)$ was activated with tris $(2$ carboxyethyl)phosphine hydrochloride (TCEP, $10 \mu \mathrm{L}$ ) as reported earlier. ${ }^{24}$ hpDNA was conjugated with small GNRs (5 $\mathrm{nM}$ ) in a molar ratio of 400:1 using a previously reported salt aging procedure. ${ }^{24,54,55}$ The conjugates were washed at $4{ }^{\circ} \mathrm{C}$ and resuspended in phosphate buffer $(\mathrm{pH} 7.5,10 \mathrm{mM}, 50 \mu \mathrm{L})$ after centrifuging.

4.6. Hybridization of hpDNA-GNRs Nanoprobes with cDNA. Excessive complimentary DNA (cDNA) was incubated with hpDNA-GNRs nanoprobes in a ratio of 1000:1 for $2 \mathrm{~h}$ at $4{ }^{\circ} \mathrm{C}$ for each sample prior to measuring the fluorescence intensity and lifetime response of the nanoprobes.

4.7. Characterization of Gold Nanorods and hpDNA GNRs. SEM (FEI Quanta FEG 250) was used for the morphological characterization of the gold nanorods using a 30 $\mathrm{kV}$ electron beam and bright field/dark field scanning transmission electron detectors. ImageJ was used for size analysis. Over 200 nanoparticles imaged at different sample 
areas were analyzed to obtain the average length and diameter. The zeta potential of the GNRs was measured using a Zetasizer Nano-ZS (Malvern Panalytical, UK).

The gold nanorods were centrifuged at $13000 \mathrm{rpm}$ for 15 min and resuspended in $2 \mathrm{~mL}$ of distilled water in a conventional plastic cuvette $(1 \mathrm{~cm}$ path length). The absorption spectra of gold nanorods were measured with a UV-visible spectrophotometer (Lambda 2, PerkinElmer). A Fluorolog spectrofluorometer (HORIBA Jobin Yvon Ltd., Middlesex, UK) was used to measure the fluorescence spectra at an excitation of $635 \mathrm{~nm}$.

Time-resolved fluorescence measurements were conducted using the time-correlated single-photon counting technique with an IBH FluoroCube fluorescence lifetime system (HORIBA Jobin Yvon IBH Ltd., Glasgow, UK). The samples were excited with a $638 \mathrm{~nm}$ pulsed light-emitting diode (NanoLED) source operating at a repetition rate of $1 \mathrm{MHz}$. Fluorescence decay measurement was taken at the magic angle $\left(54.7^{\circ}\right)$ to nullify anisotropy effects. Data analysis was performed using the DAS6 package. Fluorescence lifetimes were analyzed by fitting the decay curves to the multiexponential decay model given as

$$
I(t)=\sum_{i} B_{i} \exp \left(-\frac{t}{\tau_{i}}\right)
$$

where $\tau_{i}$ are the decay times and $B_{i}$ the associated amplitudes. The fractional contribution of each lifetime component to the steady-state intensity is represented by

$$
f_{i}=B_{i} \tau_{i} / \sum_{i} B_{i} \tau_{i}
$$

The average lifetime $(\bar{\tau})$ is calculated as.

$$
\bar{\tau}_{i}=\sum_{i} f_{i} \tau_{i}
$$

The decay curves were fitted to a 3-exponential model to account for the fluorescence decay components arising from Cy5 in hybridized and closed hpDNAs and light scattering $\left(\tau_{1}\right)$ that was fixed at 0.5 channel.

\section{ASSOCIATED CONTENT}

\section{S Supporting Information}

The Supporting Information is available free of charge on the ACS Publications website at DOI: 10.1021/acsomega.9b01200.

Preparation of growth solution to investigate the influence of silver nitrate on the size of small gold nanorods, histogram of the length and width of small gold nanorods prepared by varying the ratio of growth solution $(G)$ to seed solution, $\mathrm{G}_{6} \mathrm{~S}_{4}, \mathrm{G}_{8} \mathrm{~S}_{2}$, and $\mathrm{G}_{9} \mathrm{~S}_{1}$, histogram of the length and width of small nanorods prepared using different amounts of silver nitrate $\left(\mathrm{AgNO}_{3}, 0.01 \mathrm{M}\right)$ from 20 to $135 \mu \mathrm{L}$, UV-vis absorption spectrum of Cy5-functionalized small gold nanorods with/without cDNA, emission spectrum of Cy5 and the absorption spectra of $\mathrm{SG}_{18} \mathrm{~S}_{2}-\mathrm{CTAB}$ and $\mathrm{LG}_{18} \mathrm{~S}_{2}-\mathrm{CTAB}$, and fluorescence decay curves of $\mathrm{SG}_{18} \mathrm{~S}_{2}$ and $\mathrm{LG}_{18} \mathrm{~S}_{2}$ nanoprobes (PDF)

\section{AUTHOR INFORMATION}

\section{Corresponding Author}

*E-mail: y.chen@strath.ac.uk. Phone: +44 (0)141 5483087.

\section{ORCID 1}

Paul R. Edwards: 0000-0001-7671-7698

Yu Chen: 0000-0003-2427-3559

\section{Notes}

The authors declare no competing financial interest.

\section{ACKNOWLEDGMENTS}

Z.S.M. acknowledges a $\mathrm{PhD}$ studentship from the Federal University of Agriculture, Makurdi, Nigeria. This work was supported by a BBSRC fund (BB/K013416/1).

\section{REFERENCES}

(1) Kang, X.; Guo, X.; Niu, X.; An, W.; Li, S.; Liu, Z.; Yang, Y.; Wang, N.; Jiang, Q.; Yan, C.; Wang, H.; Zhang, Q. Photothermal therapeutic application of gold nanorods-porphyrin-trastuzumab complexes in HER2-positive breast cancer. Sci. Rep. 2017, 7, 44519.

(2) Mallick, S.; Sun, I.-C.; Kim, K.; Yi, D. K. Silica coated gold nanorods for imaging and photo-thermal therapy of cancer cells. $J$. Nanosci. Nanotechnol. 2013, 13, 3223-3229.

(3) Williams, D. Essential Biomaterials Science; Cambridge University Press: Cambridge, 2014.

(4) Hauck, T. A statistical model for estimation of gold nanorod extinction coefficients.Term report; University of Toronto, 2005.

(5) Huang, X.; El-Sayed, M. A. Gold nanoparticles: optical properties and implementations in cancer diagnosis and photothermal therapy. J. Adv. Res. 2010, 1, 13-28.

(6) Akouibaa, A.; Benhamou, M.; Derouiche, A. Simulation of the optical properties of gold nanorods : comparison to experiment. Int. J. Adv. Res. Comput. Sci. Softw. Eng. 2013, 3, 657-671.

(7) Nikoobakht, B.; El-Sayed, M. A. Surface-enhanced raman scattering studies on aggregated gold nanorods. J. Phys. Chem. A 2003, 107, 3372-3378.

(8) Zhang, Y.; Yu, J.; Birch, D. J. S.; Chen, Y. Gold nanorods for fluorescence lifetime imaging in biology. J. Biomed. Opt. 2010, 15, 020504.

(9) Sheng, Y.; Lin, M.; Li, X.; Hao, H.; Lin, X.; Sun, H.; Zhang, H. Enhancement of the $808 \mathrm{~nm}$ photothermal effect of gold nanorods by thiol-induced self-assembly. Part. Part. Syst. Charact. 2014, 31, 788793.

(10) Gu, P.; Birch, D. J. S.; Chen, Y. Dye-dope polystyrene-coated gold nanorods: towards wavelength tuneable SPASER. Methods Appl. Fluoresc. 2014, 2, 024004.

(11) Racknor, C.; Singh, M. R.; Zhang, Y.; Birch, D. J. S.; Chen, Y. Energy transfer between a biological labelling dye and gold nanorods. Methods Appl. Fluoresc. 2013, 2, 015002.

(12) Zhang, Y.; Birch, D. J. S.; Chen, Y. Two-photon excited surface plasmon enhanced energy transfer between DAPI and gold nanoparticles: opportunities in intra-cellular imaging and sensing. Appl. Phys. Lett. 2011, 99, 103701.

(13) Chen, Y.; Zhang, Y.; Birch, D. J. S.; Barnard, A. S., Creation and luminescence of size-selected gold nanorods. Nanoscale 2012, 4 (). DOI: $10.1039 / \mathrm{c} 2 \mathrm{nr} 30324 \mathrm{~h}$

(14) Zhang, Y.; Chen, Y.; Yu, J.; Birch, D. J. S. A surface plasmon enhanced FLIM-FRET imaging approach based on Au nanoparticles. Med Devices Diagn Eng 2017, 2, 78-82.

(15) Unser, S.; Bruzas, I.; He, J.; Sagle, L. Localized surface plasmon resonance biosensing: current challenges and approaches. Sensors 2015, 15, 15684-15716.

(16) Nima, Z. A.; Alwbari, A. M.; Dantuluri, V.; Hamzah, R. N.; Sra, N.; Motwani, P.; Arnaoutakis, K.; Levy, R. A.; Bohliqa, A. F.; Nedosekin, D.; Zharov, V. P.; Makhoul, I.; Biris, A. S. Targetin g nano drug delivery to cancer cells using tunable, multi-layer, silverdecorated gold nanorods. J. Appl. Toxicol. 2017, 37, 1370-1378. 
(17) Haine, A. T.; Niidome, T. Gold nanorods as nanodevices for bioimaging, photothermal therapeutics, and drug delivery. Chem. Pharm. Bull. 2017, 65, 625-628.

(18) Ali, M. R. K.; Wu, Y.; Tang, Y.; Xiao, H.; Chen, K.; Han, T.; Fang, N.; Wu, R.; El-Sayed, M. A. Targeting cancer cell integrins using gold nanorods in photothermal therapy inhibits migration through affecting cytoskeletal proteins. Proc. Natl. Acad. Sci. 2017, 114, E5655-E5663.

(19) Chen, Y.; Preece, J. A.; Palmer, R. E. processing and characterization of gold nanoparticles for use in plasmon probe spectroscopy and microscopy of biosystem. Annals of the New York Academy of Sciences 2008, 1130, 201.

(20) Mackey, M. A.; Ali, M. R. K.; Austin, L. A.; Near, R. D.; ElSayed, M. A. The Most Effective Gold Nanorod Size for Plasmonic Photothermal Therapy: Theory and In Vitro Experiments. J. Phys. Chem. B 2014, 118, 1319-1326.

(21) Sen, T.; Patra, A. Recent Advances in Energy Transfer Processes in Gold-Nanoparticle-Based Assemblies. J. Phys. Chem. C 2012, 116, 17307-17317.

(22) Zhang, Y.; Wei, G.; Yu, J.; Birch, D. J. S.; Chen, Y. Surface plasmon enhanced energy transfer between gold nanorods and fluorophores: application to endocytosis study and RNA detection. Faraday Discuss. 2015, 178, 383-394.

(23) Szmacinski, H.; Lakowicz, J. R. Fluorescence lifetime-based sensing and imaging. Sens. Actuators, B 1995, 29, 16-24.

(24) Wei, G.; Yu, J.; Wang, J.; Gu, P.; Birch, J. S. D.; Chen, Y. Hairpin DNA functionalized gold nanorods for mRNA detection in homogenous solution. J. Biomed. Opt. 2016, 21, 0097001.

(25) Broude, N. E. Stem-loop oligonucleotides: A robust tool for molecular biology and biotechnology. Trends Biotechnol. 2002, 20, 249-256.

(26) Jia, H.; Fang, C.; Zhu, X.-M.; Ruan, Q.; Wang, Y.-X. J.; Wang, J. Synthesis of absorption-dominant small gold nanorods and their plasmonic properties. Langmuir 2015, 31, 7418-7426.

(27) Ali, H. R.; Ali, M. R. K.; Wu, Y.; Selim, S. A.; Abdelaal, H. F. M.; Nasr, E. A.; El-Sayed, M. A. Gold nanorods as drug delivery vehicles for rifampicin greatly improve the efficacy of combating mycobacterium tuberculosis with good biocompatibility with the host cells. Bioconjugate Chem. 2016, 27, 2486-2492.

(28) Yue, K.; Nan, J.; Zhang, X.; Tang, J.; Zhang, X. Photothermal effects of gold nanoparticles induced by light emitting diodes. Appl. Therm. Eng. 2016, 99, 1093-1100.

(29) Ungureanu, C.; Koning, G. A.; van Leeuwen, T. G.; Manohar, $S$. The "nanobig rod" class of gold nanorods: optimized dimensions for improved in vivo therapeutic and imaging efficacy. Nanotechnology 2013, 24, 215102

(30) Shibu, E. S.; Varkentina, N.; Cognet, L.; Lounis, B. Small gold nanorods with tunable absorption for photothermal microscopy in cells. Adv. Sci. 2017, 4, 1600280.

(31) Li, Z.; Tang, S.; Wang, B.; Li, Y.; Huang, H.; Wang, H.; Li, P.; Li, C.; Chu, P. K.; Yu, X.-F. Metabolizable small gold nanorods: sizedependent cytotoxicity, cell uptake and in vivo biodistribution. ACS Biomater. Sci. Eng. 2016, 2, 789-797.

(32) Cong, B.; Kan, C.; Wang, H.; Liu, J.; Xu, H.; Ke, S. Gold Nanorods: Near-Infrared Plasmonic Photothermal Conversion and Surface Coating. J. Mater. Sci. Chem. Eng. 2014, 2, 20-25.

(33) Jiang, K.; Smith, D. A.; Pinchuk, A. Size-dependent photothermal conversion efficiencies of plasmonically heated gold nanoparticles. J. Phys. Chem. C 2013, 117, 27073-27080.

(34) Pearce, M. E.; Melanko, J. B.; Salem, A. K. Multifunctional Nanorods for Biomedical Applications. Pharm. Res. 2007, 24, 23352352.

(35) Li, Z.; Huang, H.; Tang, S.; Li, Y.; Yu, X.-F.; Wang, H.; Li, P.; Sun, Z.; Zhang, H.; Liu, C.; Chu, P. K. Small gold nanorods laden macrophages for enhanced tumor coverage in photothermal therapy. Biomaterials 2016, 74, 144-154.

(36) Warrier, P.; Yuan, Y.; Beck, M. P.; Teja, A. S. Heat transfer in nanoparticle suspensions: modeling the thermal conductivity of nanofluids. AIChE J. 2010, 56, 3243-3256.
(37) Swierczewska, M.; Lee, S.; Chen, X. The design and application of fluorophore-gold nanoparticle activatable probes. Phys. Chem. Chem. Phys. 2011, 13, 9929-9941.

(38) Gole, A.; Murphy, C. J. Seed-Mediated Synthesis of Gold Nanorods: Role of the Size and Nature of the Seed. Chem. Mater. 2004, 16, 3633-3640.

(39) Nikoobakht, B.; El-Sayed, M. A. Preparation and growth mechanism of gold nanorods (NRs) using seed-mediated growth method. Chem. Mater. 2003, 15, 1957-1962.

(40) Ali, M. R. K.; Snyder, B.; El-Sayed, M. A. Synthesis and optical properties of small Au nanorods using a seedless growth technique. Langmuir 2012, 28, 9807-9815.

(41) Burrows, N. D.; Harvey, S.; Idesis, F. A.; Murphy, C. J. Understanding the seed-mediated growth of gold nanorods through a fractional factorial design of experiments. Langmuir 2017, 33, 18911907

(42) Sau, T. K.; Murphy, C. J. Seeded high yield synthesis of short Au nanorods in aqueous solution. Langmuir 2004, 20, 6414-6420.

(43) Perezjuste, J.; Pastorizasantos, I.; Lizmarzan, L.; Mulvaney, P. Gold nanorods: Synthesis, characterization and applications. Coord. Chem. Rev. 2005, 249, 1870-1901.

(44) Park, K.; Hsiao, M.-s.; Yi, Y.-J.; Izor, S.; Koerner, H.; Jawaid, A.; Vaia, R. A. Highly concentrated seed-mediated synthesis of monodispersed gold nanorods. ACS Appl. Mater. Interfaces 2017, 9, 26363-26371.

(45) Tong, W.; Walsh, M. J.; Mulvaney, P.; Etheridge, J.; Funston, A. $M$. Control of symmetry breaking size and aspect ratio in gold nanorods: Underlying role of silver nitrate. J. Phys. Chem. C 2017, 121, 3549-3559.

(46) Zhang, Q.; Jing, H.; Li, G. G.; Lin, Y.; Blom, D. A.; Wang, H. Intertwining roles of silver Ions, surfactants, and reducing agents in gold nanorod overgrowth: pathway switch between silver underpotential deposition and gold-silver codeposition. Chem. Mater. 2016, 28, 2728-2741.

(47) Smith, D. K.; Korgel, B. A. The Importance of the CTAB surfactant on the colloidal seed-mediated synthesis of gold nanorods. Langmuir 2008, 24, 644-649.

(48) Scarabelli, L.; Sánchez-Iglesias, A.; Pérez-Juste, J.; Liz-Marzán, L. M. A "tips and tricks" practical guide to the synthesis of gold nanorods. J. Phys. Chem. Lett. 2015, 6, 4270-4279.

(49) Wang, S.; Zhao, X.; Wang, S.; Qian, J.; He, S. Biologically inspired polydopamine capped gold nanorods for drug delivery and light -mediated cancer therapy. ACS Appl. Mater. Interfaces 2016, 8, 24368-24384.

(50) Zhang, Y.; Xu, D.; Li, W.; Yu, J.; Chen, Y. Effect of size, shape, and surface modification on cytotoxicity of gold nanoparticles to human HEp-2 and canine MDCK cells. J. Nanomater. 2012, 2012, 375496.

(51) Patibandla, S.; Zhang, Y.; Tohari, A. M.; Gu, P.; Reilly, J.; Chen, Y.; Shu, X. Comparative analysis of the toxicity of gold nanoparticles in zebrafish. J. Appl. Toxicol. 2018, 38, 1153-1161.

(52) Wijaya, A.; Hamad-Schifferli, K. Ligand customization and DNA functionalization of gold nanorods via round-trip phase transfer ligand exchange. Langmuir 2008, 24, 9966-9969.

(53) Ray, K.; Zhang, J.; Lakowicz, J. R. Fluorescence lifetime correlation spectroscopic study of fluorophore-labeled silver nanoparticles. Analitical Chem. 2008, 80, 7313-7318.

(54) Hurst, S. J.; Lytton-Jean, A. K. R.; Mirkin, C. A. Maximizing DNA loading on a range of gold nanoparticle sizes. Anal. Chem. 2006, $78,8313-8318$.

(55) Wijaya, A.; Schaffer, S. B.; Pallares, I. G.; Hamad-Schifferli, K. Selective release of multiple DNA oligonucleotides from gold nanorods. ACS Nano 2009, 3, 80-86. 